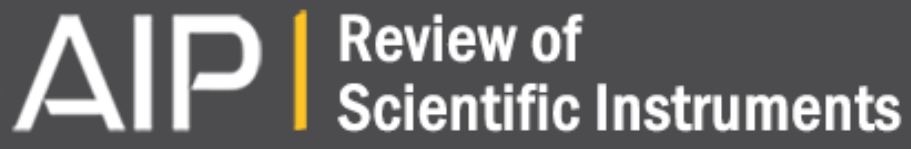

\section{A novel torsion testing technique for micro-scale specimens based on electromagnetism}

Yong Huan, Yujing Dai, Yaqi Shao, Guangjian Peng, Yihui Feng, and Taihua Zhang

Citation: Review of Scientific Instruments 85, 095106 (2014); doi: 10.1063/1.4894822

View online: http://dx.doi.org/10.1063/1.4894822

View Table of Contents: http://scitation.aip.org/content/aip/journal/rsi/85/9?ver=pdfcov

Published by the AIP Publishing

\section{Articles you may be interested in}

Simulation and testing of a micro electromagnetic energy harvester for self-powered system

AIP Advances 4, 031303 (2014); 10.1063/1.4861208

A multi-frequency sandwich type electromagnetic vibration energy harvester

Appl. Phys. Lett. 100, 213509 (2012); 10.1063/1.4722814

Electromagnetic braking: A simple quantitative model

Am. J. Phys. 74, 815 (2006); 10.1119/1.2203645

A Novel "Test Arrangement for Strain Influence on Strands" (TARSIS): Mechanical and Electrical Testing of ITER Nb3Sn Strands

AIP Conf. Proc. 711, 466 (2004); 10.1063/1.1774603

Electromagnetic cylindrical compression test under large strain at high strain rate

AIP Conf. Proc. 429, 863 (1998); 10.1063/1.55602

NEW

Model PS-100

Tabletop Cryogenic

Probe Station

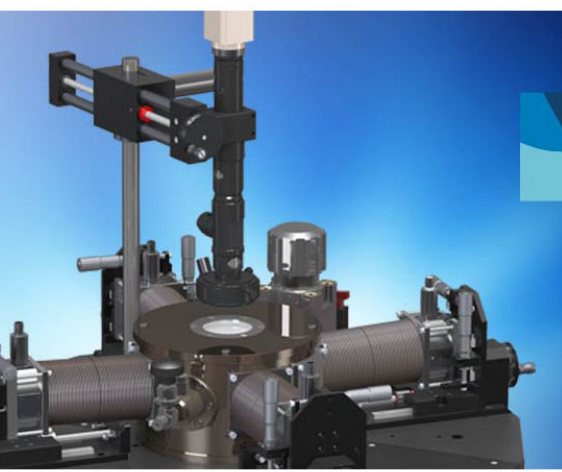

An affordable solution for a wide range of research 


\title{
A novel torsion testing technique for micro-scale specimens based on electromagnetism
}

\author{
Yong Huan, ${ }^{1, a)}$ Yujing Dai, ${ }^{1}$ Yaqi Shao, ${ }^{1}$ Guangjian Peng, ${ }^{2}$ Yihui Feng, ${ }^{1}$ and Taihua Zhang ${ }^{2}$ \\ ${ }^{1}$ State Key Laboratory of Nonlinear Mechanics (LNM), Institute of Mechanics, Chinese Academy of Sciences, \\ Beijing 100190, China \\ ${ }^{2}$ College of Mechanical Engineering, Zhejiang University of Technology, Hangzhou 310014, China
}

(Received 9 June 2014; accepted 25 August 2014; published online 12 September 2014)

\begin{abstract}
A novel torsion apparatus for micro-scale specimens is developed based on electromagnetism, in which a coil-magnet component is used for actuating and torque measuring. When the current gets through the coil, the torque, produced by Ampere force, can be easily measured by recording the current. A laser displacement sensor is applied to measure the rotation angle. The torque is calibrated using Sartorius BP211D balance. The calibration results demonstrate there is a perfect linear relationship between the torque and the current. The torque capacity is $4.0 \times 10^{-4} \mathrm{~N} \mathrm{~m}$ with noisefloor of less than $10^{-8} \mathrm{Nm}$. The rotation angle capacity is $60^{\circ}$ with noise-floor of less than $0.02^{\circ}$. Two sets of copper wire specimens, with diameter of $100 \mu \mathrm{m}$ and $140 \mu \mathrm{m}$, are tested using this apparatus. Experimental results, with good resolution and repeatability, successfully demonstrate the effectiveness of the torsion testing technique for micro-scale specimens. () 2014 AIP Publishing LLC. [http://dx.doi.org/10.1063/1.4894822]
\end{abstract}

\section{INTRODUCTION}

Torsion test is a smart and effective approach to investigate the mechanical properties of the nanocrystalline metals, ${ }^{1-3}$ metallic glasses, ${ }^{4}$ coat/matrix interfaces,${ }^{5-7}$ etc., because it can build pure shear stress conditions and the results have definite physical meanings. For macro-scale specimens with diameter of $10^{\circ} \mathrm{mm}$ or more, torsion test can be easily conducted by commercial torsion testing machine, such as MTS Bionix EM torsion test system and INSTRON MT Series Torsion Tester, etc. However, for micro-scale specimens with diameter of $10^{-1} \mathrm{~mm}$ or less, the involved torque sharply drops to $10^{-4} \mathrm{Nm}$ or even less, scaling with the cube of specimen's diameter. Commercial torsion testing machines, usually equipped with strain-gauged torque sensor, do not have enough resolution for these tests.

Probably due to the lack of suitable instruments, until now, relatively few techniques are developed for the torsion tests on micro-scale specimens. The most cited experiment was performed by Fleck et al. ${ }^{8}$ on polycrystalline copper wires in 1994. Several glass filaments were adopted as the torque sensors, which were calibrated by using a dead weight and pulley arrangement. It is necessary to replace different size glass filaments for different size specimens in order to acquire sufficient torque resolution. Because the main objective of this paper is to depict the size effects caused by a faster work hardening and not to show the torsion testing technique, some important experimental details were not described, such as the torque calibration process.

Until 2010, Lu and Song ${ }^{9}$ replicated the torsion test of micro-scale wires following the same concept of torsion experiments conducted by Fleck et al., but developed a new

a) Author to whom correspondence should be addressed. Electronic mail: huany@1nm.imech.ac.cn. Tel.: +8610 82543920. method to measure the rotation angle of micro-scale wires. Slim glass filaments were adopted to measure the torque and a laser displacement sensor is adopted to measure the rotation angle. It is a significant promotion to the torsion technique because more experiment details were reported. However, the glass filament with capacity of about $0.2 \times 10^{-3} \mathrm{~N} \mathrm{~m}$ was calibrated by commercial torque sensor with capacity of $0.5 \mathrm{Nm}$, which induced significant data scatter of up to $17 \%$. Walter and $\mathrm{Kraft}^{10}$ also developed an AFM-based torsion method with the aid of Zwick Z2.5 testing machine. Two AFM-tips were used for the torque measurement through a rigid cross-beam bonded to the specimen. For the torque calibration, a $50 \mu \mathrm{m}$ diameter tungsten filament was adopted as the reference by assuming the tungsten wire being an isotropic material of known shear modulus. This technique has a good torque resolution, but the vertical misalignment and the roughness of cross-beam may lead to an obvious data fluctuation. ${ }^{11}$ In 2011, load-unload technique was used by Dunstan et al. ${ }^{12,13}$ for studying the elastic limit and plastic deformation of thin metal wires and thin foils under torsion, tension, and bending. Plastic strain as low as $10^{-6}$ can be measured accurately with the gauge length of specimen up to $1 \mathrm{~m}$. However, the true magnitude of the torque on thin wire was not directly measured by means of this technique. In addition, Liu et al. ${ }^{14}$ reported a high-resolution torsion technique on micro-scale copper wires based on an automated torsion balance. The torque is measured by a tungsten filament with diameter of $100 \mu \mathrm{m}$, whose torsion constant was calibrated by means of a torsional vibration method. It is a smart but indirect method because the nonlinear error of the relationship between torque and rotation angle of tungsten filament cannot be presented in detail.

Almost all the above techniques followed the similar concept of macro-scale torsion testing machine, namely, a sensitive elastic element was used as torque sensor. However, it is 
difficult to combine a perfect resolution with wide torque span because the torque scales with the cube of specimen's diameter. For example, both Fleck ${ }^{8}$ and $\mathrm{Lu}^{9}$ had to replace different size glass filaments for different size specimens in order to acquire sufficient torque resolution. Moreover, the torque calibration methods were different from each other, which made the comparability and traceability of the results worse.

In this study, we abandoned the above torque measuring concept and developed a new technique based on electromagnetism, with characteristics of perfect resolution and linearity, to measure the tiny torque below $10^{-4} \mathrm{~N} \mathrm{~m}$. Finally, applications on micro-scale copper wires were presented to demonstrate the effectiveness of this technique.

\section{TEST APPARATUS}

\section{A. Design principle}

A new torsion apparatus for micro-scale specimens is developed based on electromagnetism, in which a coil placed in a radial magnetic field is used for actuating and torque measuring. The schematic diagram of the coil-magnet component is illustrated in Fig. 1. There exists a radial magnetic field between the concentric located inner and the outer magnets. The coil can rotate around the common central axis. When the current gets through the coil, the raw torque, produced by Ampere force, can be easily measured according to the following formula:

$$
T_{\text {raw }}=F w=B I l w=C I,
$$

where $F$ is Ampere force produced by one side of the coil, $w$ is the width of the coil frame, $B$ is the magnetic flux density, $I$ is the value of the current in wire, and $l$ is the total length of wire merged in magnetic field. If $B$ is constant, $C$ will be a constant, which can be determined through the beforehand calibration work. Consequently, the raw torque $T_{\text {raw }}$ can be easily measured by recording the current $I$.

In order to measure the rotation angle of the coil without any influence on the torque measurement, LX500 laser displacement sensor is applied. One fixed tape is pasted on the body frame. The other movable tape is pasted on a thin plate,

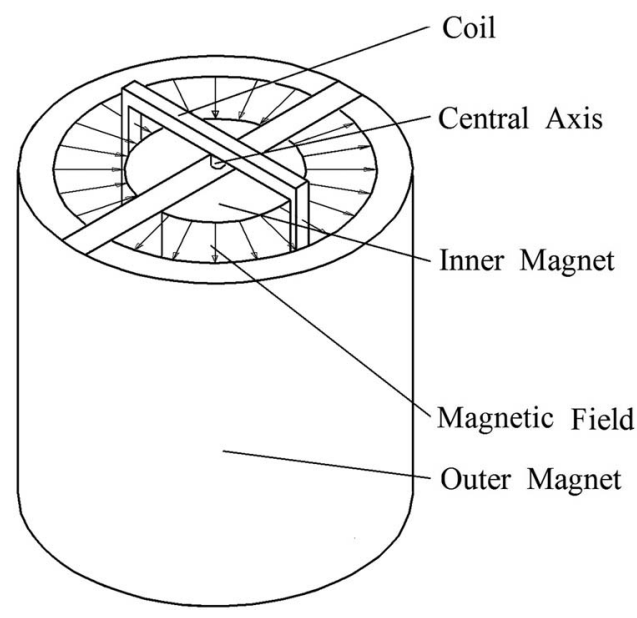

FIG. 1. Schematic diagram of the coil-magnet actuator.

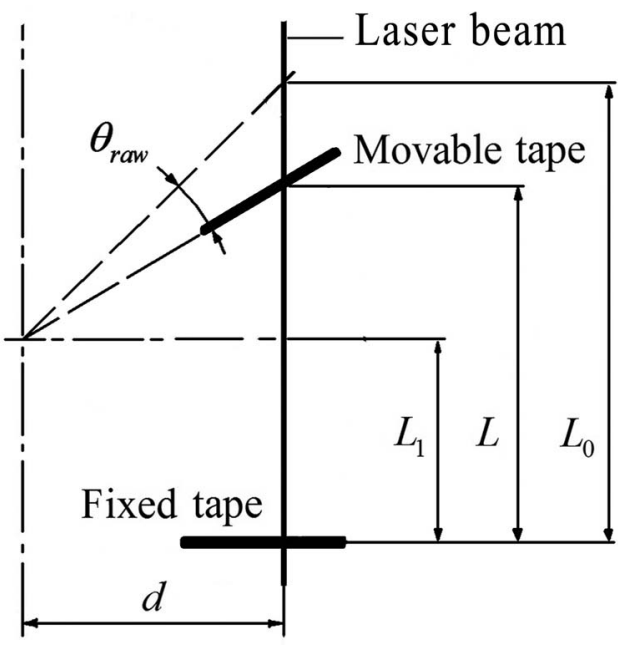

FIG. 2. Measuring principle of the raw rotation angle.

which is mounted on the coil frame. When the coil frame and the thin plate rotate together, the distance between the two reflective tapes is measured by LX500 laser displacement sensor, shown in Fig. 2. Consequently, the raw rotation angle of the coil can be calculated according to the following formula:

$$
\theta_{\text {raw }}=\arctan \left(\frac{L_{0}-L_{1}}{d}\right)-\arctan \left(\frac{L-L_{1}}{d}\right),
$$

where $d$ is the distance from the rotation center to the laser beam, $L$ is the distance between the two tapes measured by the laser displacement sensor, $L_{0}$ is the initial value of $L$, and $L_{1}$ is the distance from the rotation center to the fixed tape.

\section{B. Mechanical model}

However, after all the above components were integrated together to form the torsion apparatus, some puzzles produced. The wire must be led to a power and the coil must be supported by a bearing. Subsequently, we found that the true torque on specimen is not exactly the measured torque produced by Ampere force, and the rotation angle is not exactly the measured rotation angle. Three problems are put forward, i.e., stiffness of the lead wire, friction of the bearing, and stiffness of the body frame. In order to illustrate these influences, the mechanical model of the apparatus is shown in Fig. 3, supposing all damps are ignored in static tests.

The true torque applied on specimen $T$ and the true rotation angle of specimen $\theta$ can be written as

$$
\begin{gathered}
T=T_{\text {raw }}-T_{l}-f=T_{\text {raw }}-K_{l} \times \theta_{\text {raw }}-f, \\
\theta=\theta_{\text {raw }}-\theta_{f}=\theta_{\text {raw }}-\frac{T}{K_{f}} .
\end{gathered}
$$

From Eqs. (1) to (4), we find that the measurement reliability is influenced by the following four important problems.

First, the linear relationship $T_{\text {raw }} \propto I$ is legal only if the magnetic flux density $B$ is constant. In fact, how much does this linear relationship exist? Second, the stiffness of the lead wire will influence the measurement of the torque on 


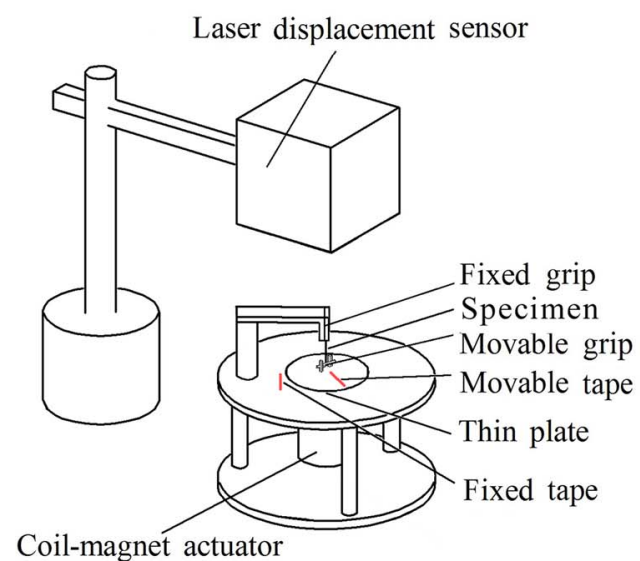

(a)

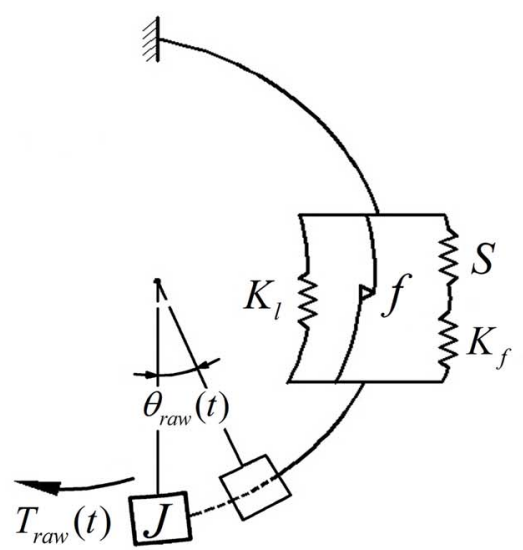

(b)

FIG. 3. Illustration of the torsion apparatus. (a) Structure diagram and (b) mechanical model. $J$-rotational inertia of the movable mass, $S$-torsional stiffness of the specimen, $K_{l}$ - torsional stiffness of the lead wire, $f$-friction torque of the bearing, and $K_{f}$-torsional stiffness of the body frame.

specimen. In order to quantitatively eliminate the influence, we assume that the stiffness of the lead wire is constant. What is the truth? Third, the friction of the bearing will also influence the measurement of the torque on specimen. How much is it? Fourth, the measurement of the specimen's rotation angle is influenced by the stiffness of the body frame. If the stiffness of the body frame is much greater than the specimen's, the influence will be very small.

In order to obtain the true torque and rotation angle of specimen according to Eqs. (3) and (4), all these problems mentioned above will be given a clear interpretation by the following calibration work.

\section{CALIBRATION}

The calibration work of torque was performed with the aid of Sartorius BP211D analytical balance, with resolution/capacity of $0.01 \mathrm{mg} / 80 \mathrm{~g}$. The torsion apparatus was placed horizontally to drive a slim and rigid probe to press the balance pan. The reading change of balance was transformed to torque by taking the length of the slim probe into account. Consequently the torque and current relationship was

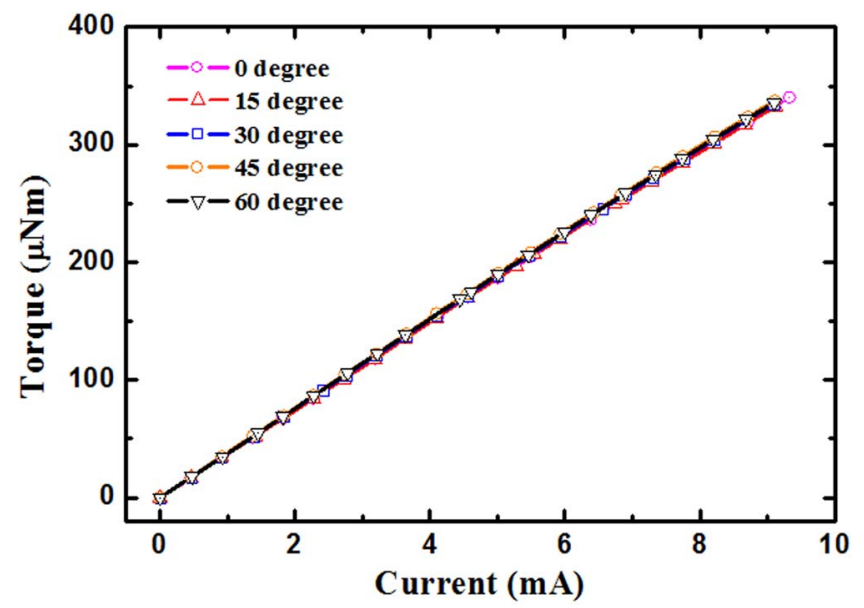

FIG. 4. Relationship between the torque and current throughout the full rotation angle capacity with intervals of $15^{\circ}$.

obtained by recording the driving current in the coil and the reading change of balance.

The relationships of the torque and current were calibrated throughout the full rotation angle capacity with intervals of $15^{\circ}$, shown in Fig. 4. A perfect linear relationship is observed between the torque and current. All the five curves coincide well and the nonlinearity is less than $1 \%$ throughout the full torque capacity. This also confirms the assumption that the magnetic flux density be constant. The torque capacity is $4.0 \times 10^{-4} \mathrm{~N} \mathrm{~m}$, which is limited by the allowable current in the coil.

The stiffness of the lead wire was calibrated by rotating the coil without any external load. The average stiffness is $4.3 \times 10^{-8} \mathrm{~N} \mathrm{~m} / \mathrm{deg}$ with nonlinearity of less than $0.5 \%$. The friction of the bearing is nearly a constant of $3.5 \times 10^{-8} \mathrm{~N} \mathrm{~m}$ at frequently used rotation speed. The stiffness of the body frame was calibrated by rotating a rigid body specimen, whose stiffness is assumed infinite. The result shows that the stiffness of the body frame is $2.0 \times 10^{-3} \mathrm{~N} \mathrm{~m} / \mathrm{deg}$, which is much greater than the specimens $\left(<10^{-5} \mathrm{~N} \mathrm{~m} / \mathrm{deg}\right)$.

Finally, the true torque and the rotation angle of specimen can be obtained after all these parameters being used in Eqs. (3) and (4).

Moreover, the noise-floor was calibrated in order to inspect the ability of the apparatus to measure the minimum signals. The torque noise-floor is below $10^{-8} \mathrm{~N} \mathrm{~m}$ and the rotation angle noise-floor is below $0.02^{\circ}$. The main technical parameters are listed in Table I.

TABLE I. Main technical parameters of this torsion apparatus.

\begin{tabular}{lc}
\hline \hline Technical parameters & Value \\
\hline Torque $(\mathrm{N} \mathrm{m})$ & \\
Capacity & $4.0 \times 10^{-4}$ \\
Noise-floor & $<10^{-8}$ \\
Rotation angle (deg) & \\
Capacity & 60 \\
Noise-floor & $<0.02$ \\
\hline \hline
\end{tabular}




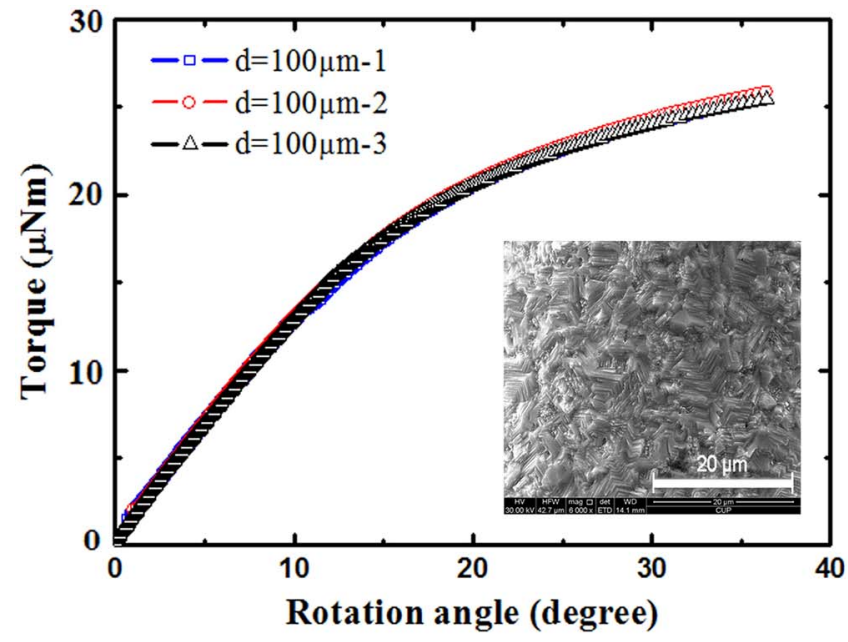

(a)

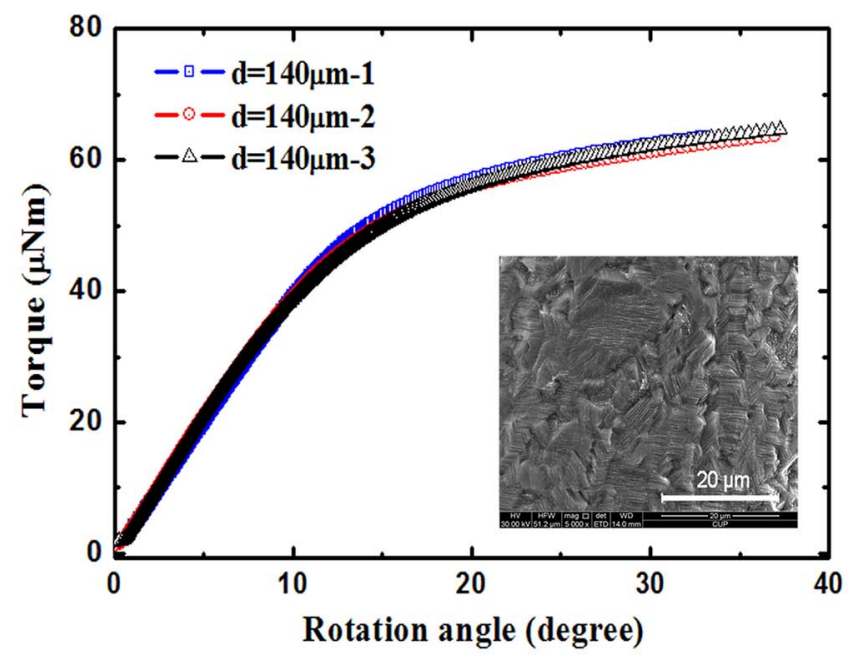

(b)

FIG. 5. Torque-rotation angle curves of wire specimens with diameter of (a) $100 \mu \mathrm{m}$ and (b) $140 \mu \mathrm{m}$. For different size specimens, the micro-textures are similar.

\section{APPLICATIONS}

In order to verify the effectiveness of the apparatus, two set of polycrystalline copper wires ( $99.9 \%$ purity) were tested, with diameter of $100 \mu \mathrm{m}$ and $140 \mu \mathrm{m}$, respectively. The exact diameter of each specimen is measured by using optical microscope before torsion test.

The copper wire specimen was bonded on a hard paperboard to avoid pre-deformation. One end of the paper-board specimen was firmly mounted on the upper grip, while the other end glued with a rigid rod was freely placed into the fork of the movable grip. The gauge length was about $5.5 \mathrm{~mm}$, separately measured after the specimen being mounted. The rod's weight provided a slight and constant tension force, with tension stress no more than 5\% yield stress of the copper, to avoid the wire specimen buckling during the test. Afterwards, the paper-board was cut off and a small torsion load was gradually applied on the wire specimen by increasing the current in the coil. The strain rate at wire's surface was of the or-

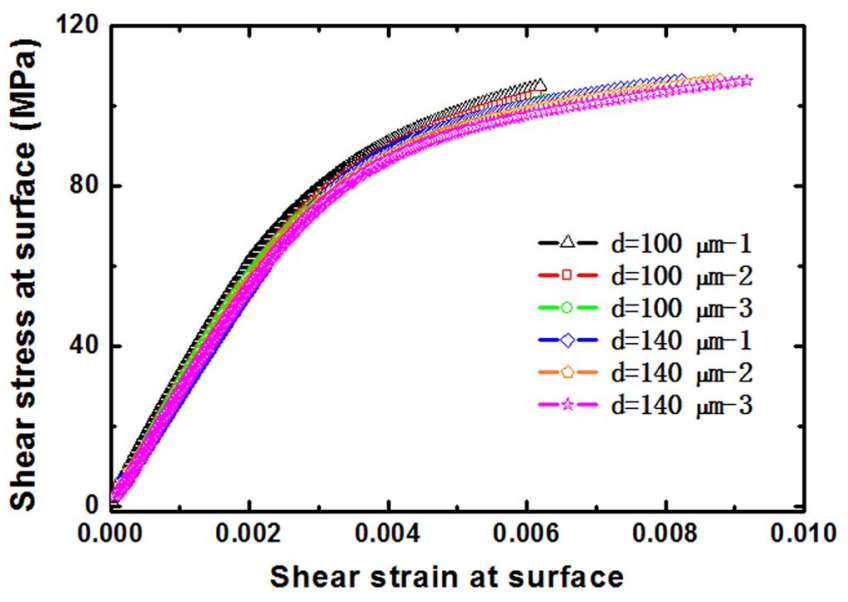

FIG. 6. Relationship between shear stress and shear strain at surface of different size specimens. The average shear modulus is $28.8 \mathrm{GPa}( \pm 6 \%)$.

der of $10^{-4} \mathrm{~s}^{-1}$. Both the raw torque and raw rotation angle were recorded by computer. The true torque and rotation angle of specimen can be calculated according to Eqs. (3) and (4), shown in Fig. 5. The micro-textures of different specimens are similar, observed in SEM.

The shear stress at surface $\tau$ and shear strain at surface $\gamma$ can be calculated according to the following equations:

$$
\begin{gathered}
\tau=\frac{16 T}{\pi D^{3}}, \\
\gamma=\frac{\theta D}{2 L_{g}},
\end{gathered}
$$

where $D$ and $L_{g}$ are the diameter and gauge length of the wire specimen, respectively.

For different size specimens, the curves of shear stressshear strain at surface present clear elastic-plastic behavior with good coincidence, shown in Fig. 6. The average shear modulus is $28.8 \mathrm{GPa}( \pm 6 \%)$, calculated from the linearity range of the curves. For different diameter specimens, no significantly different mechanical properties are observed within $0.6 \%$ shear strain.

\section{SUMMARY}

In this paper, a torsion apparatus for micro-scale specimens has been developed based on electromagnetism, in which a coil placed in radial magnetic field is used for driving and torque measuring. A laser displacement sensor is used for rotation angle measuring. The calibration results demonstrate there is a perfect linear relationship between the torque and the current. The torque capacity is $4.0 \times 10^{-4} \mathrm{~N} \mathrm{~m}$ with noise-floor of less than $10^{-8} \mathrm{~N} \mathrm{~m}$. The rotation angle capacity is $60^{\circ}$ with noise-floor of less than $0.02^{\circ}$.

Two set of copper wire specimens, with diameter of $100 \mu \mathrm{m}$ and $140 \mu \mathrm{m}$, were tested using this torsion apparatus. For different size specimens, the curves of shear stress-shear strain at surface coincide well, with average shear modulus of $28.8 \mathrm{GPa}( \pm 6 \%)$. Experimental results, with good repeatability, successfully demonstrate the effectiveness of the torsion testing technique for micro-scale specimens. 


\section{ACKNOWLEDGMENTS}

This work is supported by the National Natural Science Foundation of China (Grant Nos. 11372323, 11025212, 11272318, and 11302231).

${ }^{1}$ M. Dao, L. Lu, R. J. Asaro et al., Acta Mater. 55, 4041 (2007).

${ }^{2}$ X. Z. Liao, Y. H. Zhao, S. G. Srinivasan et al., Appl. Phys. Lett. 84, 592 (2004).

${ }^{3}$ A. V. Sergueeva, V. V. Stolyarov, R. Z. Valiev et al., Scr. Mater. 45, 747 (2001).

${ }^{4}$ Y. B. Wang, D. D. Qu, X. H. Wang et al., Acta Mater. 60, 253 (2012).

${ }^{5}$ G. F. Wang, S. W. Yu, and X. Q. Feng, Theor. Appl. Fract. Mech. 36, 195 (2001).
${ }^{6}$ B. G. Bazehhour and J. Rezaeepazhand, Appl. Compos. Mater. 18, 485 (2011).

${ }^{7}$ C. Kaya, F. Kaya, A. R. Boccaccini et al., Acta Mater. 49, 1189 (2001).

${ }^{8}$ N. A. Fleck, G. M. Muller, M. F. Ashby, and J. W. Hutchinson, Acta Metall. Mater. 42, 475 (1994).

${ }^{9}$ W. Y. Lu and B. Song, Exp. Mech. 51, 729 (2011).

${ }^{10}$ M. Walter and O. Kraft, Rev. Sci. Instrum. 82, 035109 (2011).

${ }^{11}$ D. Liu, Y. He, D. J. Dunstan et al., Int. J. Plast. 41, 30 (2013).

${ }^{12}$ D. J. Dunstan, J. U. Gallé, B. Ehrler et al., Rev. Sci. Instrum. 82, 093906 (2011).

${ }^{13}$ D. J. Dunstan, B. Ehrler, R. Bossis et al., Phys. Rev. Lett. 103, 155501 (2009).

${ }^{14}$ D. Liu, Y. He, X. Tang et al., Scr. Mater. 66, 406 (2012). 